Agron. Mesoam. 29(2):293-303. Mayo-agosto, 2018

ISSN 2215-3608, doi:10.15517/ma.v29i2.27582

http://www.revistas.ucr.ac.cr/index.php/agromeso

\title{
Respuesta de cinco leguminosas de cobertura a la fertilización fosfórica ${ }^{1}$
}

\author{
Response of five legume cover crops to phosphoric fertilization
}

\author{
Robin Gómez-Gómez², María Isabel González-Lutz ${ }^{3}$
}

\begin{abstract}
Resumen
Los cultivos de cobertura de especies leguminosas son sembradas para el control de malezas, prevención de la erosión del suelo y aporte de nitrógeno al suelo. El objetivo de este estudio fue evaluar la respuesta de las leguminosas de cobertura Mucuna pruriens cv. cinza, Mucuna pruriens cv. preta, Crotalaria spectabilis, Vigna radiata y Pueraria phaseoloides a la fertilización fosfórica, en dos épocas del año. El experimento se realizó en un invernadero en la Estación Experimental Agrícola Fabio Baudrit Moreno (EEAFBM) de la Universidad de Costa Rica, en Alajuela, Costa Rica, en el periodo de noviembre del 2012 a enero del 2013, y se repitió de abril a mayo del 2013. Se aplicaron dosis de $0,30,60$ y $90 \mathrm{~kg} \mathrm{P}_{2} \mathrm{O}_{5} / \mathrm{ha}$ en suelo esterilizado con vapor y posteriormente, utilizado para llenar potes de $2 \mathrm{~kg}$ de capacidad. Se evaluó altura de planta, número de hojas verdaderas y peso seco luego de 18-26 días de la siembra, según la especie de leguminosa. Tuvieron un mayor crecimiento $C$. spectabilis Ruth, $V$. radiata (L) Wilczek y $P$. phaseoloides (Roxb), cuando fueron fertilizadas con $60 \mathrm{~kg} \mathrm{P}_{2} \mathrm{O}_{5}$ /ha, mientras que, los dos cultivares de M. pruriens no incrementaron su crecimiento con la fertilización fosfórica. El fósforo a la siembra de las leguminosas de semilla pequeña aceleró su crecimiento, lo que indica la importancia de validar el empleo de este fertilizante para la obtención de una rápida cobertura del suelo, lo cual podría disminuir la competencia con las malezas.
\end{abstract}

Palabras clave: plantas de cobertura, manejo de malezas, abonos verdes.

\begin{abstract}
Legume cover crops are planted to control weed, to prevent soil erosion, and to increase nitrogen in soil. The objective of this study was to evaluate the response to phosphorus fertilization of the legumes Mucuna pruriens cv. cinza, Mucuna pruriens cv. preta, Crotalaria spectabilis, Vigna radiata, and Pueraria phaseoloides, during two seasons of the year. The experiment was carried out in a greenhouse at the Agricultural Experiment Station Fabio Baudrit Moreno (EEAFBM, in Spanish), of the University of Costa Rica, Alajuela, Costa Rica, from November 2012 to January 2013, and it was replicated from April to May 2013. Doses of 0,30,60, and $90 \mathrm{~kg} \mathrm{P}_{2} \mathrm{O}_{5} /$ ha were applied on steam sterilized soil, an then it was used to fill $2 \mathrm{~kg}$ capacity pots. The variables assessed were plant height, number of true leaves, and dry weight 18-26 days after planting, depending on legume species. Crotalaria spectabilis, V. radiata, and P. phaseoloides achieved the highest growth when fertilized at the rate of $60 \mathrm{~kg} \mathrm{P}_{2} \mathrm{O}_{5} /$ ha, whereas both M. pruriens cultivars did not increase growth as a response to phosphoric fertilization. Due to the positive effect of phosphorus
\end{abstract}

\footnotetext{
1 Recibido: 16 de enero, 2017. Aceptado: 12 de setiembre, 2017. Este trabajo formó parte del proyecto de investigación 736-B3-164 de la Universidad de Costa Rica (UCR), Costa Rica.

2 Universidad de Costa Rica, Estación Experimental Agrícola Fabio Baudrit Moreno. Alajuela, Costa Rica. gomez.robin@gmail.com

3 Universidad de Costa Rica, Escuela de Estadística. San José, Costa Rica. mariaisabel.gonzalezlutz@ucr.ac.cr
} 
fertilization on growth of small seeded legumes, it is important to validate the use of this fertilizer to achieve rapid soil cover, which could decrease weed competition.

Keywords: cover plants, weed control, green manures.

\section{Introducción}

Los cultivos de cobertura han sido utilizados durante décadas en Mesoamérica para el control de la erosión del suelo en laderas, aporte de materia orgánica en suelos degradados, manejo de malezas y aporte de nutrientes tales como nitrógeno y potasio (Sancho y Cervantes, 1997; Flores-Sánchez et al., 2013). Se conoce como leguminosas de cobertura a aquellas plantas de la familia Fabaceae que, por su crecimiento acelerado, su alta área foliar y su capacidad para fijar nitrógeno atmosférico en asociación con bacterias del suelo, son utilizadas como cultivos de cobertura en áreas agrícolas (Tonitto et al., 2006).

Los cultivos de cobertura cumplen muchas más funciones ecológicas dentro de los agroecosistemas, además de las ya mencionadas. La proliferación de raíces por parte de estas especies, permite una mejor infiltración del agua y mayor aireación del suelo (Lopes-Cruz et al., 2014), lo que favorece el aumento de las poblaciones microbianas benéficas (Carrera et al., 2007). Cuando los cultivos de cobertura se siembran intercalados con el cultivo, o en sus bordes, la mayor diversidad vegetal atrae organismos benéficos, tales como polinizadores, depredadores y parasitoides de insectos plaga (Altieri y Schmidt, 1986). Además, la diversidad de especies, de leguminosas que pueden ser utilizadas como cultivos de cobertura, permite al productor contar con alternativas según ciclo de vida y hábito de crecimiento, por lo que, es posible sembrarlas en diferentes áreas agrícolas, según el objetivo agronómico que se persiga.

En el mundo son muchas las especies de leguminosas cuyas características de crecimiento y tolerancia al ambiente las hacen deseables como cultivos de cobertura. Entre ellas, la especie Mucuna pruriens es una de las más utilizadas en Mesoamérica para prevenir la erosión en laderas y como abono verde (Buckles et al., 2002). Es una planta con hábito de crecimiento rastrero y trepador, que cubre muy rápido el suelo, al igual que Pueraria phaseoloides (Koutika et al., 2001). Crotalaria spectabilis por su parte, es sembrada en Sur América y Asia por su aporte de nitrógeno al suelo y también, para el control de nematodos fitopatógenos (McSorley, 1999). Vigna radiata es una especie que produce semillas comestibles, de rápido crecimiento erecto que, al alcanzar la floración se vuelve postrado, y aporta un alto contenido de nitrógeno al suelo como abono verde (Isemura et al., 2012).

El establecimiento inicial de las leguminosas de cobertura es un paso crítico del cual dependerá el éxito de su implementación, y por lo tanto, requiere de ciertas prácticas agrícolas básicas que podrían favorecer su germinación y crecimiento. Dentro de estas prácticas agrícolas determinantes está la fertilización, específicamente la adición de fósforo durante la siembra (Divito y Sadras, 2014).

Las leguminosas que adquieren el nitrógeno por fijación biológica son más sensibles a deficiencias en fósforo en comparación con aquellas que dependen del nitrógeno presente en el suelo (Israel, 1987). El papel del fósforo en estas plantas está relacionado con la formación, el crecimiento y el funcionamiento de los nódulos donde se fija el nitrógeno, debido a que, la enzima nitrogenasa requiere de una alta síntesis de ATP para su funcionamiento (Al-Niemi et al., 1998). Cuando existe un déficit de fósforo en la planta se reduce su crecimiento aéreo, debido a una reacción regulada por la concentración de nitrógeno, que modera el crecimiento y la actividad de los nódulos (Divito y Sadras, 2014). Algunas especies de leguminosas, sin embargo, tienen una mayor tolerancia al déficit de fósforo en sus primeras etapas de desarrollo, ya que éste puede provenir de las reservas contenidas en las semillas (Hoveland et al., 1976). 
El objetivo de este estudio fue evaluar la respuesta de las leguminosas de cobertura, Mucuna pruriens cv. cinza, Mucuna pruriens cv. preta, Crotalaria spectabilis, Vigna radiata, Pueraria phaseoloides a la fertilización fosfórica, en dos épocas del año.

\section{Materiales y métodos}

El experimento se realizó en un invernadero en la Estación Experimental Agrícola Fabio Baudrit Moreno (EEAFBM) de la Universidad de Costa Rica, en Alajuela, Costa Rica, en dos épocas: de noviembre del 2012 a enero del 2013 y de abril a mayo del 2013.

Se evaluaron las leguminosas Mucuna pruriens cv. cinza, Mucuna pruriens cv. preta, Crotalaria spectabilis (crotalaria), Vigna radiata (vigna) y Pueraria phaseoloides (kudzú), las cuales se sembraron en potes de plástico de dos litros de capacidad. Las semillas de los dos cultivares de mucuna se escarificaron cortando con bisturí una porción de la cubierta seminal, mientras que, las semillas de crotalaria y kudzú se escarificaron sumergiéndolas por dos minutos en agua a una temperatura de $70{ }^{\circ} \mathrm{C}$.

El suelo utilizado fue previamente esterilizado con vapor de agua a $100{ }^{\circ} \mathrm{C}$ por treinta minutos, para eliminar patógenos, insectos y semillas de malezas en dormancia, según la metodología reportada por Castillo-Luna y Gómez-Gómez (2016). El análisis físico-químico del suelo utilizado, realizado después de la esterilización, se presenta en el Cuadro 1.

Cuadro 1. Contenido de nutrientes, $\mathrm{pH}$ y acidez del suelo empleado en la evaluación del efecto de la fertilización fosfórica sobre leguminosas de cobertura. Alajuela, Costa Rica. 2016.

Table 1. Nutrient content, $\mathrm{pH}$ and acidity of soil used for the evaluation of phosphorus fertilization effect on five legume cover crops. Alajuela, Costa Rica. 2016.

\begin{tabular}{|c|c|c|c|c|c|c|c|c|c|}
\hline & $\mathrm{Ca}$ & & $\mathbf{K}$ & $\mathbf{P}$ & $\mathbf{Z n}$ & & $\mathrm{Fe}$ & Mn & Relación \\
\hline pH $\mathrm{H}_{2} \mathrm{O}$ & & $\operatorname{mol}(+$ & & & 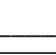 & ng/l & - & & $\mathrm{C} / \mathrm{N}$ \\
\hline 5,9 & 9,01 & 2,59 & 0,35 & 2 & 0,7 & 9 & 85 & 85 & 13,5 \\
\hline
\end{tabular}

Se utilizó fosfato monoamónico (MAP, 12-61-0) como fuente fosfórica. Las dosis de $\mathrm{P}_{2} \mathrm{O}_{5}$ evaluadas fueron 0, 30, 60 y $90 \mathrm{~kg} / \mathrm{ha}$. Se aplicó también urea (46-0-0) con el fin de uniformizar en $20 \mathrm{~kg} / \mathrm{ha}$ la dosis de nitrógeno en cada tratamiento, ya que, el MAP contiene también nitrógeno. Para calcular las cantidades de fertilizante por adicionar, se consideró una densidad de siembra de 40000 plantas por hectárea.

El diseño del experimento fue completamente al azar. Debido a que, cada leguminosa tiene hábito de crecimiento diferente, se analizaron los datos de manera separada para cada especie. Las variables evaluadas en plantas de crotalaria y vigna, y las dos especies de mucuna, fueron altura de planta (desde el suelo hasta el punto de crecimiento apical), número de hojas verdaderas y peso seco; mientras que, en plantas de Pueraria se evaluó número de hojas verdaderas y peso seco. Las plantas de mucuna, crotalaria, vigna y kudzú, se cortaron a los 18 , 22,26 y 20 días después de la siembra, respectivamente, y se secaron en estufa a $65{ }^{\circ} \mathrm{C}$ por 72 horas. La diferencia en el momento de corta se debió al tipo de crecimiento de cada especie.

El modelo estadístico utilizado para crotalaria, vigna y kudzú, fue un arreglo factorial de dos factores: época del experimento a dos niveles y dosis continua de $\mathrm{P}_{2} \mathrm{O}_{5}$, con el fin de determinar si la tendencia de cada variable respuesta se da por igual en ambas épocas. Con este arreglo se probaron tres hipótesis: (1) el promedio de la 
variable respuesta es el mismo en las dos épocas (efecto simple); (2) existe una tendencia lineal en la variable respuesta al aumentar la dosis de $\mathrm{P}_{2} \mathrm{O}_{5}$ (efecto simple); (3) la tendencia de la variable respuesta se da por igual en las dos épocas (interacción).

Para mucuna el arreglo factorial fue de tres factores: época del experimento, especie y dosis continua. En este caso hay una hipótesis para cada efecto simple y tres para interacciones dobles. Lo que se busca cuando se repite un experimento en diferentes épocas, es determinar si los resultados que se obtienen para el o los efectos que se están estudiando se sostienen al cambiar las condiciones en las que se estudian. Es deseable, entonces, que tanto el efecto época como su interacción con la dosis sean no significativos, o, lo que es lo mismo, que no haya efecto.

Las variables de respuesta analizadas para cada una de las leguminosas fueron: el área bajo la curva para la altura (ABC altura), el área bajo la curva para el número de hojas verdaderas (ABC hojas), obtenidas ambas a partir de las cuatro mediciones que se hicieron en el tiempo, y el peso seco por planta. Para probar las hipótesis se usó un nivel de significancia del 5\%. Los análisis respectivos se efectuaron con el programa JMP (SAS Institute, 2015).

\section{Resultados}

Se determinó un efecto positivo de la fertilización fosfórica en las leguminosas crotalaria, kudzú y vigna en las dos épocas estudiadas. Las probabilidades asociadas a las pruebas de ambos efectos en crotalaria, vigna y pueraria, fueron superiores al 5\%, nivel fijado para declarar una prueba significativa (Cuadro 2). El efecto época no significativo indicó que, los promedios del área bajo la curva de la altura y de las hojas, así como el de peso seco, fueron iguales en ambas épocas. Las interacciones época*dosis no significativas indicaron que, la tendencia lineal, al aumentar la dosis, del ABC altura y del ABC hojas, así como la del peso seco, se dio por igual en ambas épocas. Tomando en cuenta estos resultados, se procedió a analizar la tendencia de cada una de las variables respuesta ante

Cuadro 2. Probabilidades asociadas a las pruebas de hipótesis de los efectos simples y la interacción para cada una de las variables de respuesta estudiadas en Crotalaria spectabilis, Vigna radiata y Phaseolus phaseoloides, al aplicarse dosis crecientes de $\mathrm{P}_{2} \mathrm{O}_{5}(0,30,60$ y 90 kg/ha). Alajuela, Costa Rica. 2016.

Table 2. Probabilities associated to simple effect hypothesis tests and to the interaction for each of the response variables for Crotalaria spectabilis, Vigna radiata, and Phaseolus phaseoloides treated with increasing rates of $\mathrm{P}_{2} \mathrm{O}_{5}(0,30,60$, and 90 kg/ha). Alajuela, Costa Rica. 2016.

\begin{tabular}{lccc}
\hline $\begin{array}{l}\text { Variable respuesta por } \\
\text { leguminosa }\end{array}$ & $\begin{array}{c}\text { Efecto } \\
\text { simple época }\end{array}$ & $\begin{array}{c}\text { Efecto lineal } \\
\text { de la dosis }\end{array}$ & $\begin{array}{c}\text { Interacción } \\
\text { época x dosis }\end{array}$ \\
\hline $\boldsymbol{C}$. spectabilis & & & \\
ABC altura & 0,6176 & 0,2967 & 0,1400 \\
ABC hojas & 0,7963 & 0,0030 & 0,5384 \\
Peso por planta & 0,8722 & 0,0010 & 0,6020 \\
$\boldsymbol{V}$. radiata & & & \\
ABC altura & 0,5294 & 0,0015 & 0,0590 \\
ABC hojas & 0,2373 & $<0,001$ & 0,7691 \\
Peso por planta & 0,3567 & 0,0002 & 0,9358 \\
P. phaseoloides & & & 0,4410 \\
ABC hojas & 0,9803 & 0,0002 & 0,6675 \\
Peso por planta & 0,8002 & $<0,001$ & \\
\hline
\end{tabular}


aumentos sucesivos en la dosis de $\mathrm{P}_{2} \mathrm{O}_{5}$ en cada una de las leguminosas, que resultó ser significativa para todas, con excepción del $\mathrm{ABC}$ altura en crotalaria (Cuadro 2).

El comportamiento de las variables evaluadas en las plantas de crotalaria se ajustó mejor a una función cuadrática de la dosis que a una función lineal, especialmente para el ABC altura, razón por la cual, el efecto lineal resultó no significativo (Figura 1). Esta característica permitió escoger de forma aproximada la dosis que maximiza la respuesta a la dosis de fertilizante en cada caso. Asimismo, la función cuadrática explicó de mejor manera el
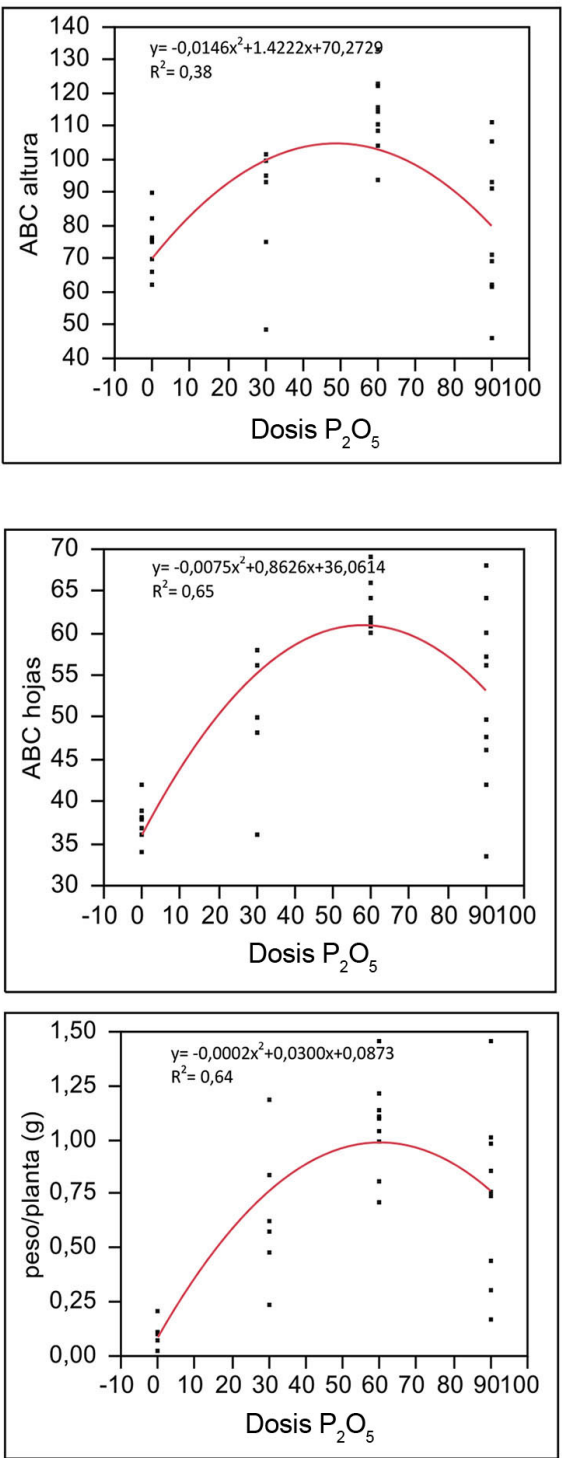

Figura 1. Tendencias cuadráticas por variable respuesta en Crotalaria spectabilis aplicada con dosis crecientes de $\mathrm{P}_{2} \mathrm{O}_{5}$. Alajuela, Costa Rica. 2016.

Figure 1. Quadratic tendency by response variable for Crotalaria spectabilis treated with increasing rates of $\mathrm{P}_{2} \mathrm{O}_{5}$. Alajuela, Costa Rica. 2016. 
comportamiento de las variables $\mathrm{ABC}$ hojas y peso por planta de vigna (Figura 2). En cuanto a la variable $\mathrm{ABC}$ hojas de kudzú, el ajuste lineal tuvo un porcentaje de variancia explicada por el modelo $\left(\mathrm{R}^{2}\right)$ muy bajo, mientras que, el peso seco presentó un ajuste cuadrático (Figura 3).
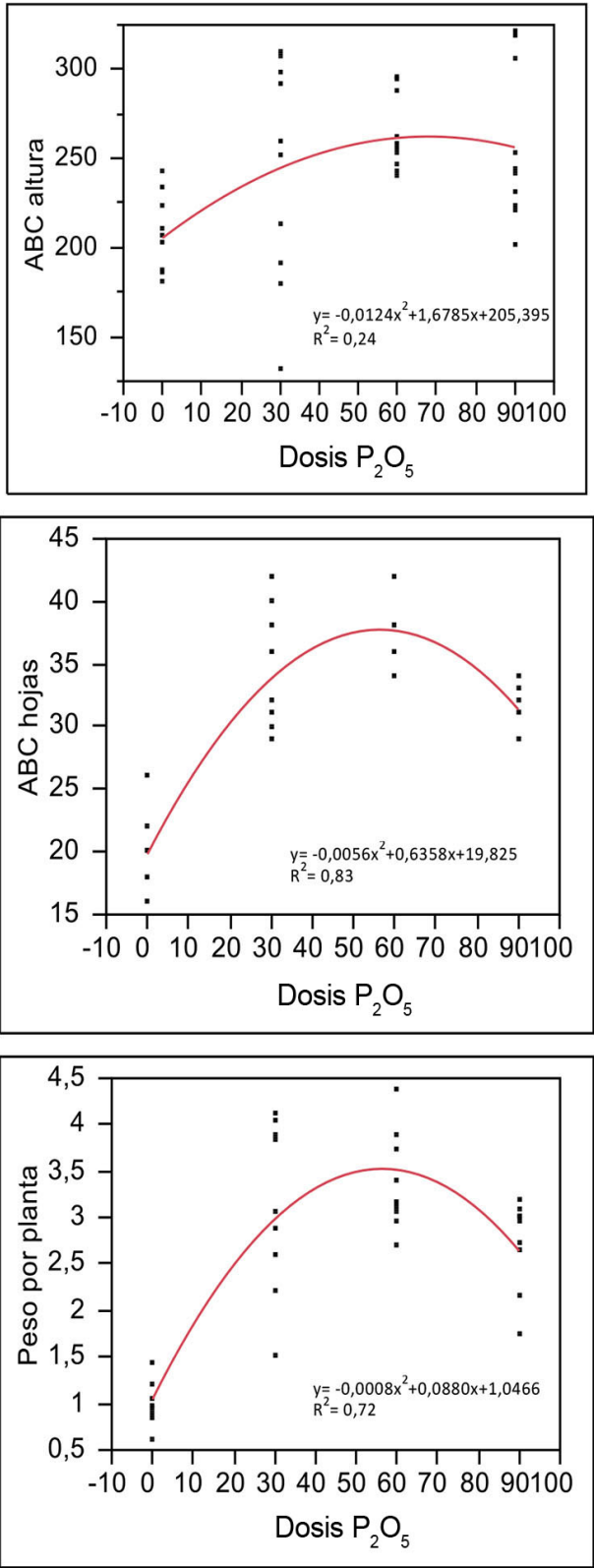

Figura 2. Tendencia cuadrática por variable respuesta en Vigna radiata aplicada con dosis crecientes de $\mathrm{P}_{2} \mathrm{O}_{5}$. Alajuela, Costa Rica. 2016.

Figure 2. Quadratic tendency by response variable for Vigna radiata treated with increasing rates of $\mathrm{P}_{2} \mathrm{O}_{5}$. Alajuela, Costa Rica. 2016. 

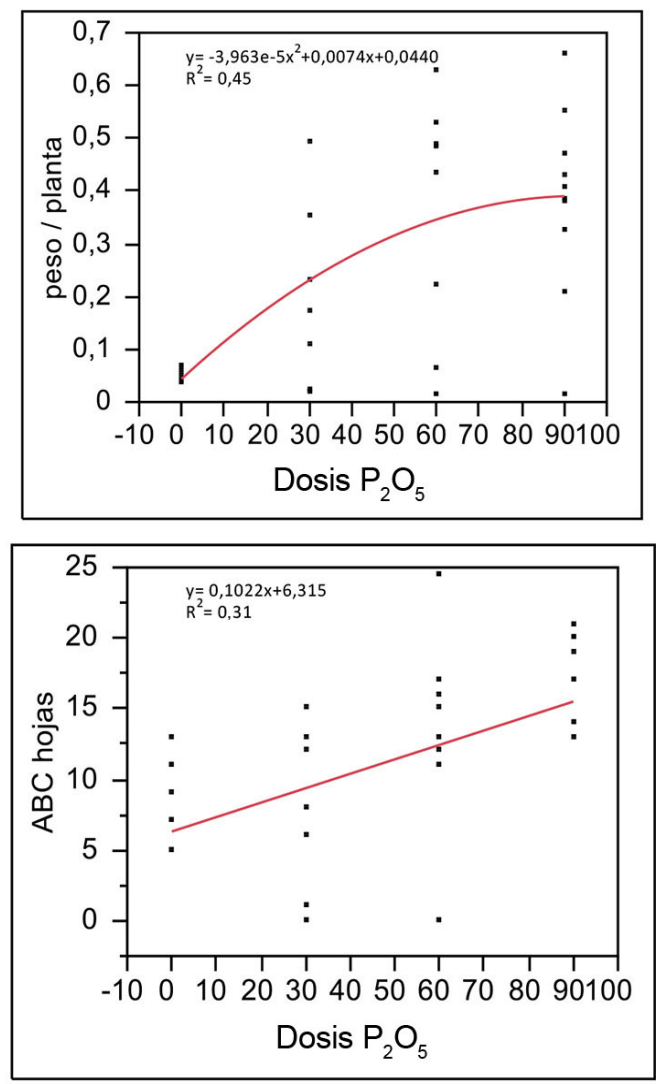

Figura 3. Tendencia cuadrática y lineal por variable respuesta en Phaseolus phaseoloides aplicada con dosis crecientes de $\mathrm{P}_{2} \mathrm{O}_{5}$. Alajuela, Costa Rica. 2016.

Figure 3. Lineal and quadratic tendency by response variable for Phaseolus phaseoloides treated with increasing rates of $\mathrm{P}_{2} \mathrm{O}_{5}$. Alajuela, Costa Rica. 2016.

Con respecto a los dos cultivares de mucuna, además del efecto época y el efecto dosis, y su respectiva interacción, se incluyó una comparación entre ellos y las respectivas interacciones con época y dosis, para las tres variables respuesta estudiadas (Cuadro 3). Solamente resultaron significativas la comparación de los promedios del ABC altura y su interacción con la época, y la interacción del ABC hojas con la época. Esto implica que, el crecimiento de cada una de las especies presentó un promedio diferente en cada una de las épocas estudiadas. Aplicar fertilizante en cualquiera de sus dosis no tuvo efecto alguno ni en el crecimiento ni en el peso en ninguna de las dos especies. Para ambas especies no se dio una tendencia lineal, como tampoco cuadrática al aumentar la dosis de fertilizante. El Cuadro 4 muestra, para el ABC altura y el $\mathrm{ABC}$ hojas, un crecimiento promedio mayor en abril-mayo para ambas especies, pero el incremento porcentual en ambas variables fue superior en la M. pruriens cv. cinza (66,5\% y $22,5 \%$, respectivamente). 
Cuadro 3. Probabilidades asociadas a las pruebas de hipótesis de los efectos simples y las interacciones para cada una de las variables respuesta estudiadas en Mucuna pruriens cv. preta y Mucuna pruriens cv. Cinza, al aplicarse dosis crecientes de $\mathrm{P}_{2} \mathrm{O}_{5}(0$, 30, 60 y 90 kg/ha). Alajuela, Costa Rica. 2016.

Table 3. Probabilities associated to simple effect hypothesis tests and to the interactions for each of the response variables for Mucuna pruriens cv. Preta, and Mucuna pruriens cv. cinza treated with increasing rates of $\mathrm{P}_{2} \mathrm{O}_{5}(0,30,60$, and $90 \mathrm{~kg} / \mathrm{ha})$. Alajuela, Costa Rica. 2016.

\begin{tabular}{lccc}
\hline Efecto & \multicolumn{3}{c}{ Variable respuesta } \\
\cline { 2 - 4 } & ABC altura & ABC hojas & Peso por planta \\
\hline Mucuna & $<0,001$ & 0,0205 & 0,3275 \\
Época & $<0,001$ & 0,0002 & 0,1089 \\
Dosis & 0,5847 & 0,9067 & 0,1117 \\
Época*cultivar & $<0,001$ & 0,0205 & 0,3580 \\
Dosis*cultivar & 0,2738 & 0,2279 & 0,5354 \\
Dosis*época & 0,0661 & 0,4128 & 0,4499 \\
\hline
\end{tabular}

Cuadro 4. Promedios de área bajo la curva $(\mathrm{ABC})$ para la altura y $\mathrm{ABC}$ para hojas para Mucuna pruriens cv. preta y Mucuna pruriens cv. cinza, por época, aplicadas con dosis crecientes de $\mathrm{P}_{2} \mathrm{O}_{5}(0,30,60$ y $90 \mathrm{~kg} / \mathrm{ha})$ para analizar la interacción. Alajuela, Costa Rica. 2016.

Table 4. Mean values of area under the curve (ABC) for height and $\mathrm{ABC}$ leaves for Mucuna pruriens cv. preta and Mucuna pruriens cv. cinza by season, treated with increasing rates of $\mathrm{P}_{2} \mathrm{O}_{5}(0,30,60$, and $90 \mathrm{~kg} / \mathrm{ha})$ to analyze the interaction. Alajuela, Costa Rica. 2016.

\begin{tabular}{lcccc}
\hline \multirow{2}{*}{ Época } & \multicolumn{2}{c}{ ABC altura } & \multicolumn{2}{c}{ ABC \# hojas } \\
\cline { 2 - 5 } & M. pruriens cv. cinza & M. pruriens cv. preta & M. pruriens cv. cinza & M.pruriens cv. preta \\
\hline Nov. 2012-Ene. 2013 & 71,47 & 78,62 & 6,20 & 7,55 \\
Abr.-May. 2013 & 213,19 & 144,52 & 8,00 & 8,00 \\
\hline
\end{tabular}

\section{Discusión}

En este estudio, el área bajo la curva de la altura, el número de hojas y el peso por planta de crotalaira fue máximo con la dosis de $60 \mathrm{~kg} / \mathrm{ha}$ de $\mathrm{P}_{2} \mathrm{O}_{5}$; al aumentar la dosis a $90 \mathrm{~kg} / \mathrm{ha}$ el valor de tales variables disminuyó en comparación con la dosis anterior. Esto indica que, el crecimiento aéreo de crotalaria aumentó con la adición de fertilización fosfórica. De manera similar, Fageria et al. (2015) encontraron una respuesta positiva de crotalaria a la fertilización fosfórica, y además, reportaron un incremento en la eficiencia del uso de zinc, cobre y hierro en las plantas fertilizadas con fósforo en comparación con aquellas no fertilizadas. La eficiencia en el uso del hierro reportada por estos autores, aumentó con la dosis de fósforo aplicada, pero presentó también una tendencia cuadrática como la mostrada en la Figura 1. Los resultados obtenidos por Hoveland et al. (1976), confirman que crotalaria, aunque tolerante a deficiencias de fósforo en el suelo, aumenta su crecimiento relativo con la aplicación de este elemento al suelo. Con base en el estudio de Rosolem et al. (2002), el crecimiento radical de crotalaria no se afectó por la compactación moderada del suelo (hasta 1,34 MPa/cm), lo que, le permite la absorción eficiente de macronutrientes tales como el fósforo.

Un comportamiento similar a crotalaria mostró la leguminosa vigna. Se observó un efecto positivo en el crecimiento de estas plantas hasta alcanzar un máximo con la dosis de $60 \mathrm{~kg} / \mathrm{ha}$. Varios estudios confirman que 
esta leguminosa es muy susceptible a la fertilización fosfórica (Gunawardena et al., 1992; Nadeem et al., 2004; Chaudhary et al., 2008), pero la respuesta encontrada en varios de ellos fue lineal y no cuadrática. Esto podría estar relacionado con los diferentes genotipos evaluados en cada estudio. En Asia, vigna es un alimento común y ha sido sometida a mejoramiento genético por muchos años, por lo que, existen gran cantidad de genotipos y su comportamiento agronómico puede variar entre sí (Isemura et al., 2012). Aun así, la dosis recomendada en tales estudios varía entre 46 y $90 \mathrm{~kg} / \mathrm{ha}$ de $\mathrm{P}_{2} \mathrm{O}_{5}$.

La respuesta de kudzú a la fertilización fosfórica fue muy variable dentro de cada dosis de $\mathrm{P}_{2} \mathrm{O}_{5}$ aplicada. En general, se observó una respuesta positiva a la aplicación de MAP, principalmente al considerar el peso por planta. El aumento en esta variable es deseable, puesto que, una mayor biomasa aérea está relacionada con mayor fijación de nitrógeno (Divito y Sadras, 2014). Un efecto similar, aumento de la biomasa al aplicar fósforo, fue observado en un estudio realizado en plantas de esta leguminosa inoculadas con micorrizas específicas (Waidyanatha et al., 1979; Salinas et al., 1985). La inoculación de micorrizas vesículo-arbusculares, favorece la absorción del fósforo por parte de kudzú en comparación con plantas sin inocular (Waidyanatha et al., 1979). La respuesta positiva a la fertilización fosfórica también ocurre en plantas sin inoculación previa (Cadisch et al., 1989).

Contrario a las otras especies de leguminosas evaluadas, los dos cultivares de mucuna no respondieron significativamente a la fertilización fosfórica, su desarrollo fue similar con o sin adición de MAP, aun cuando el contenido inicial de fósforo en el suelo fue muy bajo $(2 \mathrm{mg} / \mathrm{ml})$. De manera similar, Hairiah et al. (1995) no encontraron diferencias en el crecimiento en el perfil del suelo de raíces de mucuna cuando las plantas se trataron con diferentes dosis de fósforo, y Carsky et al. (2001) determinaron que, el crecimiento de mucuna no se incrementó al aplicar dosis bajas de fósforo ( $<18 \mathrm{~kg} / \mathrm{ha}$ ). En ciertos países de África, sin embargo, se aplican dosis moderadas de fósforo (menos de $50 \mathrm{~kg} / \mathrm{ha}$ ) a los barbechos con mucuna para mejorar la eficiencia de la fijación de nitrógeno de la leguminosa y así, suplir de una mayor cantidad de nitrógeno orgánico al cultivo siguiente, que por lo general, es un cereal (Kihara et al., 2010).

\section{Conclusiones}

Los resultados de este estudio respaldan la hipótesis de que las leguminosas de semillas pequeñas presentan una mayor respuesta a la fertilización fosfórica en comparación con las especies de semillas más grandes, que tienen mayores reservas de este elemento (Kolawole y Kang, 1997). Esta respuesta, sin embargo, podría ser mayor o menor según las condiciones edáficas y climáticas de cada sitio donde se desee sembrar estas leguminosas, por lo que, es necesario diseñar estrategias de manejo acordes con el sitio donde se establecerán los cultivos de cobertura.

\section{Literatura citada}

Al-Niemi, T.S., M.L. Kahn, and T.R. McDermott. 1998. Phosphorus uptake by bean nodules. Plant Soil 198:71-78. doi:10.1023/A:1004200903458

Altieri, M.A., and L.L. Schmidt. 1986. Cover crops affect insect and spider populations in apple orchards. Calif. Agric. 40:1517.

Buckles, D., B. Triomphe, y G. Sain. 2002. Los cultivos de cobertura en la agricultura de laderas: innovación de los agricultores con Mucuna. CIID, CIMMYT, y CATIE, CAN.

Cadisch, G., R. Sylvester-Bradley, and J. Nösberger. 1989. 15N-Based estimation of nitrogen fixation by eight tropical foragelegumes at two levels of P:K supply. Field Crops Res. 22:181-194. doi:10.1016/0378-4290(89)90091-9 
Carrera, L.M., J.S. Buyer, B. Vinyard, A.A. Abdul-Baki, L.J. Sikora, and J.R. Teasdale. 2007. Effects of cover crops, compost, and manure amendments on soil microbial community structure in tomato production systems. Appl. Soil Ecol. 37:247255. doi:10.1016/j.apsoil.2007.08.003

Carsky, R.J., B. Oyewole, and G. Tian. 2001. Effect of phosphorus application in legume cover crop rotation on subsequent maize in the savanna zone of West Africa. Nutr. Cycl. Agroecosyst. 59:151-159. doi:10.1023/A:1017578628062

Castillo-Luna, M.P., y R. Gómez-Gómez. 2016. Efecto de la esterilización del suelo con vapor de agua sobre semillas de malezas. Agron. Mesoam. 27:1-5. doi:10.15517/am.v27i2.21286

Chaudhary, M.I., J.J. Adu-Gyamfi, H. Saneoka, N.T. Nguyen, R. Suwa, S. Kanai, H.A. El-Shemy, D.A. Lightfoot, and K. Fujita. 2008. The effect of phosphorus deficiency on nutrient uptake, nitrogen fixation and photosynthetic rate in mashbean, mungbean and soybean. Acta Physiol. Plant 30:537-544. doi:10.1007/s11738-008-0152-8

Divito, G.A., and V.O. Sadras. 2014. How do phosphorus, potassium and sulphur affect plant growth and biological nitrogen fixation in crop and pasture legumes? A meta-analysis. Field Crops Res. 156:161-171. doi:10.1016/j.fcr.2013.11.004

Fageria, N.K., E.P. de-Brito-Ferreira, and A. Moreira. 2015. Micronutrients use efficiency in tropical cover crops as influenced by phosphorus fertilization. Rev. Caatinga 28:130-137.

Flores-Sanchez, D., A. Pastor, E.A. Lantinga, W.A.H. Rossing, and M.J. Kropff. 2013. Quelle Agroecol. Sustain. Food Syst. 37:739-761. doi:10.1080/21683565.2013.763888

Gunawardena, S.F.B.N., S.K.A. Danso, and F. Zapata. 1992. Phosphorus requirements and nitrogen accumulation by three mungbean (Vigna radiata (L) Welzek) cultivars. Plant Soil 147:267-274. doi:10.1007/BF00029078

Hairiah, K., M. van-Noordwijk, and S. Setijono. 1995. Tolerance and avoidance of Al toxicity by Mucuna pruriens var. utilis at different levels of P supply. Plant Soil 171:77-81. doi:10.1007/BF00009568

Hoveland, C.S., G.A. Buchanan, and M.C. Harris. 1976. Response of weeds to soil phosphorus and potassium. Weed Sci. 24:194-201.

Isemura, T., A. Kaga, S. Tabata, P. Somta, P. Srinives, T. Shimizu, U. Jo, D.A. Vaughan, and N. Tomooka. 2012. Construction of a genetic linkage map and genetic analysis of domestication related traits in mungbean (Vigna radiata). PLoS ONE 7(8):e41304. doi:10.1371/journal.pone.0041304

Israel, D.W. 1987. Investigation of the role of phosphorus in symbiotic dinitrogen fixation. Plant Physiol. 84:835-840.

Kihara, J., B. Vanlauwe, B. Waswa, J. Kimetu, J. Chianu, and A. Bationo. 2010. Strategic phosphorus application in legumecereal rotations increases land productivity and profitability in Western Kenya. Exp. Agric. 46:35-52. doi:10.1017/ S0014479709990810

Kolawole, G.O., and B.T. Kang. 1997. Effect of seed size and phosphorus fertilization on growth of selected legumes. Commun. Soil Sci. Plant Anal. 28:1223-1235. doi:10.1080/00103629709369868

Koutika, L.S., S. Hauser, and J. Henrot. 2001. Soil organic matter assessment in natural regrowth, Pueraria phaseoloides and Mucuna pruriens fallow. Soil Biol. Biochem. 33:1095-1101. doi:10.1016/S0038-0717(01)00015-3

Lopes-Cruz, J.L., L. da-Silva-Souza, N.C. dos-Santos-de-Souza, and C.R. Pelacani. 2014. Effect of cover crops on the aggregation of a soil cultivated with papaya (Carica papaya L.). Sci. Hortic. 172:82-85. doi:10.1016/j.scienta.2014.03.045

McSorley, R. 1999. Host suitability of potential cover crops for root-knot nematodes. J. Nematol. 31:619-623.

Nadeem, M.A., R. Ahmad, and M.S. Ahmad. 2004. Effect of seed inoculation and different fertilizer levels on the growth and yield of mungbean (Vigna radiata L.). J. Agron. 3:40-42. doi:10.39237ja.2004.4042 
Rosolem, C.A., J.S.S. Foloni, and C.S. Tiritan. 2002. Root growth and nutrient accumulation in cover crops as affected by soil compaction. Soil Tillage Res. 65:109-115. doi:10.1016/S0167-1987(01)00286-0

Salinas, J.G., J.I. Sanz, and E. Sieverding. 1985. Importance of VA mycorrhizae for phosphorus supply to pasture plants in tropical Oxisols. Plant Soil 84:347-360. doi:10.1007/BF02275474

Sancho, F., y C. Cervantes. 1997. El uso de plantas de cobertura en sistemas de producción de cultivos perennes y anuales en Costa Rica. Agron. Costarricense 21(1):111-120.

SAS Institute. 2015. The JMP system for Windows. Release 12. SAS Institute, Cary, NC, USA.

Tonitto, C., M.B. David, and L.E. Drinkwater. 2006. Replacing bare fallows with cover crops in fertilizer-intensive cropping systems: A meta-analysis of crop yield and N dynamics. Agric. Ecosyst. Environ. 112:58-72. doi:10.1016/j. agee.2005.07.003

Waidyanatha, U.P.d.S., N. Yogaratnam, and W.A. Ariyaratne. 1979. Mycorrhizal infection on growth and nitrogen fixation of Pueraria and Stylosanthes and uptake of phosphorus from two rock phosphates. New Phytol. 82:147-152. doi:10.1111/j.1469-8137.1979.tb07569.x 